\title{
The Influence of Kidney Stones and Salivary Uric Acid on Dental Calculus Formation and Periodontal Status among Some Saudi Patients Aged 25 - 70 Years
}

\author{
Mohammed M. A. Abdullah Al-Abdaly ${ }^{1 *}$, Fayez Saud Thlaab Alharbi², Ali Mohammed Almoalem², \\ Nasser Ahmed Taib Awaji ${ }^{2}$
}

${ }^{1}$ Periodontics and Community Dental Sciences Department, College of Dentistry, King Khalid University, Abha, Saudi Arabia

${ }^{2}$ College of Dentistry, King Khalid University, Abha, Saudi Arabia

Email: ^malabdaly20@gmail.com

How to cite this paper: Al-Abdaly, M.M.A.A., Alharbi, F.S.T., Almoalem, A.M. and Awaji, N.A.T. (2020) The Influence of Kidney Stones and Salivary Uric Acid on Dental Calculus Formation and Periodontal Status among Some Saudi Patients Aged 25 - 70 Years. International Journal of Clinical Medicine, 11, 565-578.

https://doi.org/10.4236/ijcm.2020.1110049

Received: September 25, 2020

Accepted: October 19, 2020

Published: October 22, 2020

Copyright $\odot 2020$ by author(s) and Scientific Research Publishing Inc. This work is licensed under the Creative Commons Attribution International License (CC BY 4.0).

http://creativecommons.org/licenses/by/4.0/

\begin{abstract}
Background: The formation of kidney stones is considered a complicated process. Consequently, there are many questions about the link between kidney stones formation and level of salivary uric acid and calculus formation on the teeth surfaces. Objectives: To evaluate the correlation between the level of salivary uric acid and kidney stones formation and their influence on dental calculus and periodontal status among Saudi patients aged 25 - 70 years. Materials and Methods: 120 Saudi male patients were examined (60 of Kidney stones patients and 60 patients of non-kidney stones patients) for clinical evaluation of plaque index (PLI), gingival index (GI), calculus index of oral hygiene (CI) and clinical attachment loss (CAL). Moreover, lab assessment of uric acid level in the collected salivary samples was done. The findings were analyzed using of ANOVA test and Tukey's test. Results: There were statistically significant differences in clinical parameters among kidney stones patients and non-kidney stones patients $(\mathrm{p}<0.05)$, but these differences were highly statistically significant in the correlation between calculus index (CI), plaque index (PLI) and gingival index (GI) among kidney stone patients in group II, moreover, PLI and clinical attachment loss (CAL) among kidney stone patients in group III $(\mathrm{p}<0.001)$. The statistical analyses revealed statistically significant differences in the level of salivary uric acid $(\mathrm{mg} / \mathrm{dl})$ in the comparison between kidney stones patients and non-kidney stones patients in group I and group III, whereas there were highly statistically significant in the comparison between kidney stones patients and non-kidney stones patients in group III. Conclusion: At the end of this study, we concluded that
\end{abstract}


there was a relationship between dental calculus formation, kidney stones formation, and an increase in the level of salivary uric acid.

\section{Keywords}

Dental Calculus, Kidney Stones, Uric Acid, Periodontal Status

\section{Introduction}

The oral cavity is a part of the body that includes various microorganisms, which subsist as a normal community of complex biofilms, structurally organized and multispecies [1] [2]. Therefore, it may be an effective method for the assessment of general and oral health [3].

On the other hand, Saliva is the main liquid in the oral cavity and is essential to keep the oral tissues in healthy status by inhibiting proliferation specific microorganisms, and moreover, inhibiting the build-up of dental plaque and dental calculus [4]. Consequently, saliva may be considered a mirror that can reflect the normal and abnormal states of the body [3].

The anti-oxidative system in saliva includes different enzymes and molecules which are periodontal biomarkers in the evaluation of the periodontal status [5] [6]. Uric acid participates in the antioxidant ability of saliva and blood. Further, it forms nearly $70 \%-85 \%$ of this ability in the saliva of healthy and periodontitis patients [7] [8] [9]. According to previous studies, there was a possible effect of uric acid and other antioxidants in periodontal disease initiation and progression in addition to uric acid association with some systemic abnormality such as kidney stones formation [10] [11].

A kidney stone is one of the most prevalent systemic diseases of current communities and infects $12 \%-15 \%$ of the population, and calcium oxalate stones are considered the dominant type of kidney stones worldwide [12] [13] [14]. The possibility of kidney stones recurrence is approximately $10 \%-50 \%$ through 1 - 5 years from the first infects of kidney stones [15]. In Saudi Arabia, the dehydration and hot weather are reflecting on the estimation of kidney stone risk on the life where the kidney stone incident is at least 50\% more than the western countries and, up to $90 \%$ of these patients exhibit abnormal oral changes [16] [17].

Dental calculus is the mineralization of dental plaque on the natural tooth surfaces and dental prosthesis in the mouth. Moreover, it has the same biological system of the structure formation process of kidney stones and according to the place of calculus, there are supra and subgingival calculus that are covered by unmineralized dental plaque which is the main etiologic factor of periodontal diseases [18] [19] [20] due to the sharing of kidney stones and dental calculus in the process of calcification and formation factors. Also, there was no prior Saudi study found carrying out among patients from Aseer region to evaluate the influence of kidney stones and salivary uric acid on dental calculus formation and 
periodontal status among some Saudi patients aged 25 - 70 years. Therefore, the present study was designed for the assessment of these objectives [21].

\section{Materials and Methods}

\subsection{Ethical Clearance}

The current retrospective study carried out at the outpatients' clinics, college of dentistry, King Khalid University between March 2020 G and August 2020 G. The research proposal was approved by the scientific research committee, college of dentistry, King Khalid University. The objectives of the study were fully exhibited to the patients at the beginning of this study, and the patients' informed consent was obtained to fulfill ethical clearance (IRB/KKUCOD/ETH/2019-20/039). The sample size of the study was identified according to the number of patients who come to the outpatients' clinics, the college of dentistry, King Khalid University through the study duration. The $\mathrm{G}^{\star}$ Power software program was used for performing the sample size calculation [22].

The patients' data related to the patient name, age, gender, and general health were collected from the medical files of the patients. Furthermore, we asked the patients about oral hygiene measures, bad oral habits, and the presence or absence of xerostomia.

\subsection{Participant Selection}

According to the proposal of this study, a total of 120 Saudi males patients interviewed 60 patients (study group) diagnosed as kidney stones patients based on radiographs and general urine investigations, and 60 patients were diagnosed without kidney stones based on ultrasound in the latter three months (control group). All patients were examined clinically to evaluate the amount of dental calculus and periodontal status; moreover, the salivary uric acid level was measured. The patients were divided according to the ages into three equal groups (n = 40): (Group I) $25-40$ years, (Group II) $41-55$ years, and (Group III) $56-70$ years. All groups included two equal subgroups $(n=20)$. The subgroups comprised 20 kidney stones patients as study subgroups and 20 healthy patients (non-kidney stones) as the control subgroup.

\subsection{Inclusion and Exclusion Criteria}

The patients of the study group were without other systemic diseases and, the size of the kidney stone was $20 \mathrm{~mm}$. They were using tooth brushing but did not visit the dental clinics for calculus removal since one year ago. They did not wear orthodontic appliances or fixed or removable dental prostheses. Moreover, they were in a fasting status, whereas the patients of the control group were of good medical status.

The exclusion criteria were the patients who received periodontal therapy within the past 6 months, the patients with bone disease, endocrine disease, blood diseases, medically compromised patients, alcoholic patients, the patients with vitamin D deficiency, and who need calcium supplements. The comprehensive 
kidney function evaluation was obtained from patients' medical reports that were within the patients' files to exclude any patients with other kidney diseases.

\subsection{Clinical Examination}

Periodontal examination was done by the assessment of plaque index (PLI) [23], gingival index (GI) [24], calculus index of oral hygiene index (CI) [25] and, clinical attachment loss (CAL). All clinical parameters were recorded by a Williams probe with a diameter of $0.5 \mathrm{~mm}$ (Hu-FriedyIns Co., USA) of each tooth except third molar teeth. PLI, GI, and CI were assessed on mesio-buccal, buccal, disto-buccal, and lingual or palatal, whereas CAL was assessed in interdental areas according to the last classification of periodontal diseases [26] [27] [28]. PLI was recorded before the collection of saliva samples. The clinical findings were recorded in particular case sheets and charts.

\subsection{Salivary Collection of Saliva and Biochemical Investigations}

The samples of un-stimulated saliva were obtained in the morning after breakfast $(9-11 \mathrm{am})$ to reduce circadian changes. The patients were demanded to swallow of saliva and stay stable $10 \mathrm{~min}$ for collection of saliva into a sterilized plastic. The samples were transported on dry ice within less than $30 \mathrm{~min}$ for lab investigation after stored at $-70^{\circ} \mathrm{C}$. The experiment of saliva collection was conducted by the Modified Navazesh method [29]. A centrifuge was used at $4000 \mathrm{~g}$ for 10 minutes at room temperature to remove cell debris, and an Erba kit was applied as an enzymatic method to identify uric acid.

\subsection{Statistical Analysis}

Statistical analyses of this study were done by ANOVA test for an account and, demonstrate the mean and standard deviation of the clinical results, and biochemical investigations findings. The scale of significance was assessed by using Tukey's test, and it was acceptable at $\mathrm{P}<0.05$, and highly significant when $\mathrm{P}<0.01$.

\section{Results}

The present study included a total of 120 male Saudi patients (50\% kidney stones patients and 50\% non-kidney stones patients) into three different age groups. The distribution of patients as kidney stones patients or non-kidney stones patients within the study groups as subgroups, which appears in Table 1 and Figure 1. Table 1 and Figure 1 illustrate the age distribution and the mean of age among study groups. The mean age for non-kidney stones patients was $32.01 \mathrm{ys}$ and 31.22 ys for kidney stones patients in group I. Moreover, it was 49.3 ys for non-kidney stones patients and 51.1 ys for kidney stones patients in group II, whereas it was 63.51 ys for non-kidney stones patients and 65.87 ys for kidney stones patients in group III. There were statistically significant differences in the ages between groups I, II, and III ( $\mathrm{p}<0.05)$ but, there were no statistically significant differences in the age between non-kidney stones patients and kidney stones patients in this study $(\mathrm{p}>0.05)$. 
Table 1. Age distribution and the mean of age among study groups.

\begin{tabular}{|c|c|c|c|c|c|c|c|c|c|}
\hline \multirow{2}{*}{ Groups } & \multirow{2}{*}{ Range } & \multirow{2}{*}{ No } & \multirow{2}{*}{\multicolumn{2}{|c|}{ Mean \pm SD }} & \multicolumn{2}{|c|}{ ANOVA } & \multicolumn{3}{|c|}{ Tukey's test } \\
\hline & & & & & $\mathrm{F}$ & $\mathrm{P}$-value & I\&II & I\&III & II\&III \\
\hline \multirow[b]{2}{*}{ GI } & \multirow[b]{2}{*}{$25-40$ ys } & \multirow[b]{2}{*}{40} & $\mathrm{~N}-\mathrm{KS}$ & $32.01 \pm 8.11$ & \multirow{6}{*}{ 4. 322} & \multirow{6}{*}{0.031} & \multirow{6}{*}{0.021} & \multirow{6}{*}{0.013} & \multirow{6}{*}{0.040} \\
\hline & & & KS & $31.22 \pm 4$ & & & & & \\
\hline \multirow{2}{*}{ G II } & \multirow[b]{2}{*}{$41-55$ ys } & \multirow{2}{*}{40} & N-KS & $49.3 \pm 2.46$ & & & & & \\
\hline & & & KS & $51.1 \pm 4.21$ & & & & & \\
\hline \multirow{2}{*}{ G III } & \multirow{2}{*}{$56-70$ ys } & \multirow{2}{*}{40} & N-KS & $63.51 \pm 6.32$ & & & & & \\
\hline & & & KS & $65.87 \pm 9.71$ & & & & & \\
\hline N-KS & $25-70$ ys & 60 & & $27 \pm 5.63$ & & & & & \\
\hline KS & $25-70$ ys & 60 & & $39 \pm 5.97$ & 1.712 & 0.100 & & 0.505 & \\
\hline
\end{tabular}

G: group, No: Number of participants, SD: Standard deviation, N-KS: non-Kidney stones, KS: Kidney stones.

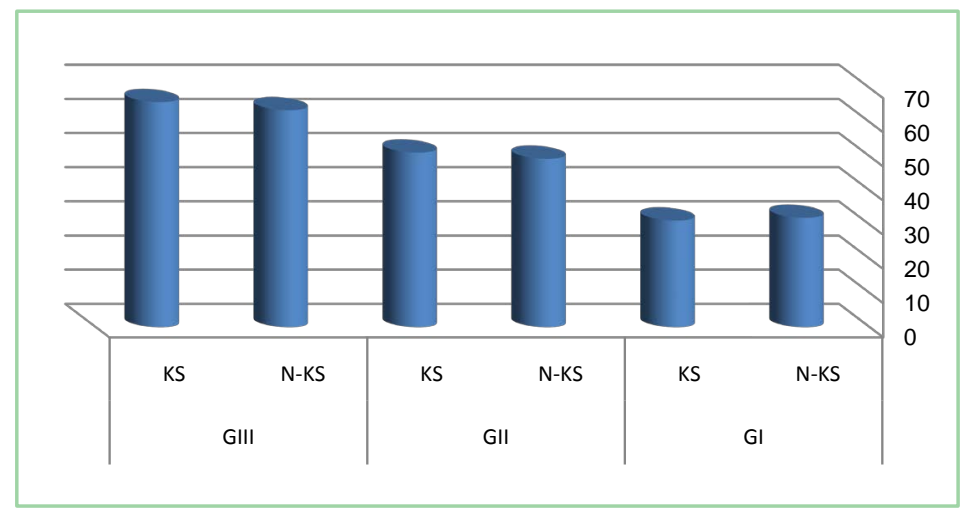

Figure 1. The mean of age among the study groups. G: group, N-KS: non-Kidney stones, KS: Kidney stones.

When we asked the patients about the self-plaque control, the feeling of dry mouth, and bad oral habits, just $45 \%$ of kidney stones patients reported that they used a toothbrush in self plaque control compared to $55 \%$ of non-kidney stones patients. Moreover, $87 \%$ of kidney stones patients were feeling of dry mouth and $13 \%$ of non-kidney stones patients clarified that there were no feeling of dry mouth. Opposite we detected in the patients' answers that $78 \%$ of patients were smokers (50\% kidney stones patients and 50\% non-kidney stones patients).

Regarding the comparison of groups I, II and, II in clinical parameter values. There were statistically significant differences in PLI and CI among the kidney stone patients plus GI and CI among non-kidney stones patients $(\mathrm{p}<0.05)$, whereas there were no statistically significant differences in GI and CAL among the kidney stones patients moreover PLI and CAL among non-kidney stones patients ( $p>0.05$ ) (Table 2 and Figure 2).

On the other hand, there were statistically significant differences in PLI, GI, $\mathrm{CI}$ and CAL in the comparison between group I, II the kidney stones patients ( $\mathrm{p}$ $<0.05$ ) vice versa in non-kidney stones patients where there were no statistically 
significant differences in PLI, GI, CI, and CAL ( $\mathrm{p}>0.05)$ and also there were statistically significant differences between group I and III in PLI, GI, CI, and CAL among kidney stones patients moreover, PLI and GI among non-kidney stones patients $(\mathrm{p}<0.05)$, whereas we did not find statistically significant differences in CI and CAL among non-kidney stones patients ( $p>0.05$ ). Furthermore, there were no statistically significant differences between group II and III in all clinical parameters except CI among non-kidney stones patients ( $\mathrm{p}>0.05)$.

In this study, the correlation between clinical parameters with CI of all patients was analyzed by ANOVA test (Table 3). Significant statistically significant differences between clinical parameters and CI were observed among kidney stones patients and non-kidney stones patients $(\mathrm{p}<0.05)$, but these differences were highly statistically significant in the correlation between CI and PLI and GI among kidney stones patients in group II moreover, PLI and CAL among kidney stones patients in group III $(\mathrm{p}<0.001)$.

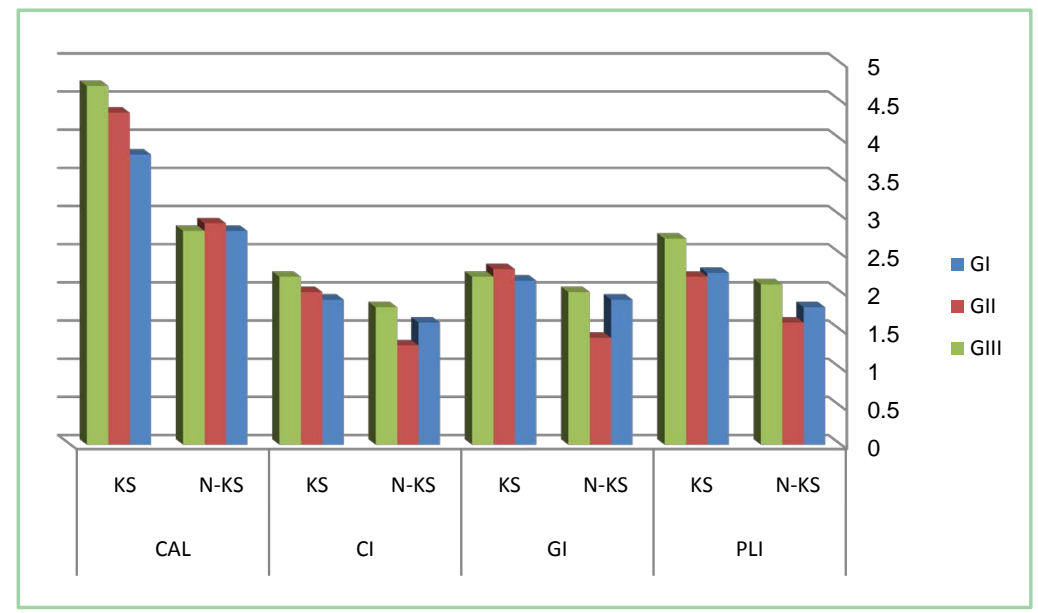

Figure 2. Clinical findings. PLI: Plaque index, GI: Gingival index, CI: Calculus index, CAL: Clinical attachment loss.

Table 2. Clinical findings.

\begin{tabular}{|c|c|c|c|c|c|c|c|c|c|}
\hline & \multirow{2}{*}{$\mathrm{CP}$} & \multicolumn{3}{|c|}{ Groups } & \multicolumn{2}{|c|}{ ANOVA } & \multicolumn{3}{|c|}{ Tukey's test } \\
\hline & & GI & GII & GIII & $\mathrm{F}$ & $\mathrm{P}$-value & I\&II & I\&III & II\&III \\
\hline \multirow{2}{*}{ PLI } & $\mathrm{N}-\mathrm{KS}$ & $1.8 \pm 0.2$ & $1.6 \pm 0,6$ & $2.1 \pm 0.4$ & 2.322 & 0.115 & 0.112 & 0.001 & 0.279 \\
\hline & KS & $2.25 \pm 1.2$ & $2.2 \pm 0.7$ & $2.7 \pm 1.1$ & 1.499 & 0.044 & 0.011 & 0.002 & 0.232 \\
\hline \multirow{2}{*}{ GI } & $\mathrm{N}-\mathrm{KS}$ & $1.9 \pm 0.6$ & $1.4 \pm 0.5$ & $2 \pm 0.3$ & 1.014 & 0.050 & 0.498 & 0.003 & 0.081 \\
\hline & KS & $2.15 \pm 0.4$ & $2.3 \pm 0.9$ & $2.2 \pm 0.4$ & 1.55 & 0.722 & 0.001 & 0.006 & 0.356 \\
\hline \multirow{2}{*}{ CI } & $\mathrm{N}-\mathrm{KS}$ & $1.6 \pm 0.2$ & $1.3 \pm 0.6$ & $1.8 \pm 0.4$ & 1.662 & 0.000 & 0.077 & 0.336 & 0.014 \\
\hline & KS & $1.9 \pm 0.4$ & $2.0 \pm 05$ & $2.2 \pm 0.4$ & 3.618 & $0.001^{*}$ & 0.004 & 0.000 & 0.315 \\
\hline \multirow{2}{*}{ CAL } & $\mathrm{N}-\mathrm{KS}$ & $2.8 \pm 1.6$ & $2.9 \pm 1.5$ & $2.8 \pm 1.8$ & 1.148 & 0.863 & 0.133 & 0.622 & 0.091 \\
\hline & KS & $3.8 \pm 1.2$ & $4.35 \pm 0.7$ & $4.7 \pm 0.6$ & 4.47 & 0.465 & 0.033 & 0.000 & 0.420 \\
\hline
\end{tabular}

PLI: Plaque index, GI: Gingival index, CI: Calculus index, CAL: Clinical attachment loss. 
Table 3. Correlation in clinical parameters with calculus index of all patients with ANOVA test.

\begin{tabular}{|c|c|c|c|c|c|c|c|c|}
\hline \multirow{2}{*}{ Groups } & \multirow{2}{*}{$\mathrm{CP}$} & \multirow{2}{*}{ PSG } & \multicolumn{2}{|c|}{ PLI } & \multicolumn{2}{|c|}{ GI } & \multicolumn{2}{|c|}{ CAL } \\
\hline & & & $\mathbf{r}$ & P-value & $\mathbf{r}$ & P-value & $\mathbf{r}$ & P-value \\
\hline \multirow[b]{2}{*}{ GI } & \multirow{2}{*}{$\mathrm{CI}$} & N-KS & 0.573 & 0.035 & 0.724 & 0.040 & 0.818 & 0.036 \\
\hline & & KS & 0.276 & 0.015 & 0.229 & 0.021 & 0.481 & 0.041 \\
\hline \multirow{2}{*}{ GII } & \multirow{2}{*}{ CI } & N-KS & 0.602 & 0.007 & 0.811 & 0.011 & 0.516 & 0.013 \\
\hline & & KS & 0.335 & 0.001 & 0.298 & 0.000 & 0.169 & 0.022 \\
\hline \multirow{2}{*}{ GIII } & \multirow{2}{*}{ CI } & N-KS & 0.246 & 0.023 & 0.447 & 0.036 & 0.691 & 0.024 \\
\hline & & KS & 0.899 & 0.001 & 0.399 & 0.003 & 0.188 & 0.001 \\
\hline
\end{tabular}

G: Group, PLI: Plaque index, GI: Gingival index, CI: Calculus index, CAL: Clinical attachment loss.

Table 4 and Figure 3 demonstrate the mean of salivary uric acid levels among the patients in the different groups $(\mathrm{mg} / \mathrm{dl})$ of the present study. The statistical analyses revealed statistically significant differences in the level of salivary uric acid in the comparison between kidney stones patients and non-kidney stones patients in group I and group III, whereas there were highly statistically significant in the comparison between kidney stones patients and non-kidney stones patients in group III.

The correlation between age and all clinical parameters were summarized in Table 5. There were statistically significant differences in the comparison between the age of patients and all clinical parameters except GI.

Regarding the correlation between the level of salivary uric acid and all clinical parameters, it is found to be statistically significant differences in the comparison between the level of salivary uric acid with PLI and GI whereas it was highly statistically significant in the comparison between the level of salivary uric acid with CI and CAL (Table 6).

\section{Discussion}

Several studies showed the calcifications in the body (stones) with a decrease in the number of studies that tried to demonstrate the connection between these stones. These studies clarified that these stones have the same structure. Therefore, some of the studies considered their formation as metabolic disorders, and their components are identical in different organs [30] [31] [32]. In this retrospective study, the dental calculus formation was detected among most kidney stones patients more than non-kidney stones patients due to the kidneys' work in an important way to organize the levels of uric acid in serum [33]. Similar to the study results of Davidovich E. et al. where they demonstrated that the calcium, phosphate, uric acid, and magnesium components in saliva play an important role in the association between kidney stones formation and, dental calculus formation. Furthermore, they found there was a relationship between the distribution of mineral metabolism and calculus formation among kidney disease patients [34]. 


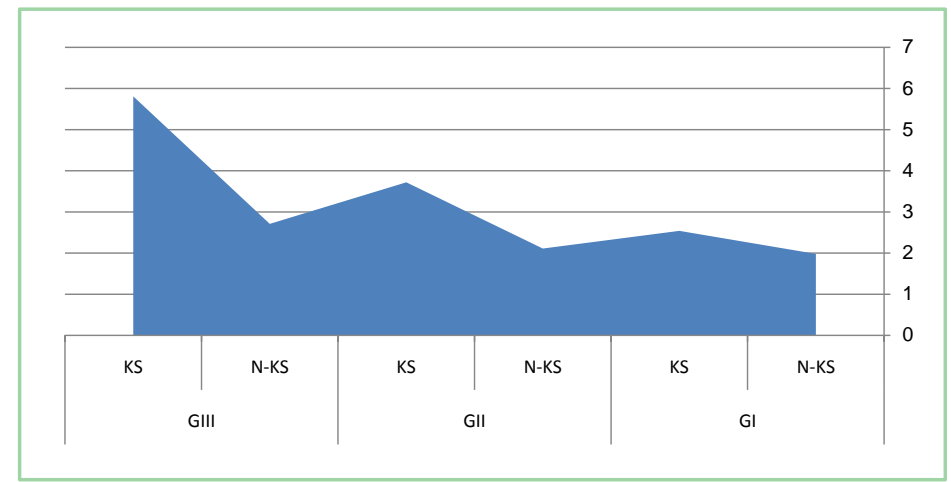

Figure 3. Salivary uric acid level. G: group, N-KS: non-Kidney stones, KS: Kidney stones.

Table 4. The mean of salivary uric acid level among patients in different groups (mg/dl).

\begin{tabular}{ccccccr}
\hline \multirow{2}{*}{ Groups } & & Mean \pm SD & \multicolumn{5}{c}{ ANOVA } \\
\cline { 5 - 7 } Group I & N-KS & $1.98 \pm 0.43$ & F & P-value & F & P- value \\
\hline \multirow{2}{*}{ Group II } & KS & $2.54 \pm 0.67$ & 0.615 & 0.051 & & \\
& N-KS & $2.11 \pm 0.31$ & & & 0.302 & 0.001 \\
Group III & N-KS & $2.72 \pm 0.70$ & 0.798 & 0.002 & & \\
& KS & $5.81 \pm 0.52$ & & & & \\
\hline
\end{tabular}

G: group, SD: Standard deviation, N-KS: non-Kidney stones, KS: Kidney stones.

Table 5. The Correlation between Age and all parameters.

\begin{tabular}{ccc}
\hline \multirow{2}{*}{ Correlations } & \multicolumn{2}{c}{ Age } \\
\cline { 2 - 3 } & $\mathrm{r}$ & P-value \\
\hline PLI & 0.233 & $0.005^{*}$ \\
GI & 0.062 & 0.433 \\
CI & 0.03 & $0.003^{*}$ \\
CAL & 0.177 & $0.05^{*}$ \\
\hline
\end{tabular}

PLI: Plaque index, GI: Gingival index, CI: Calculus index, CAL: Clinical attachment loss. *statistically significant differences.

Table 6. The Correlation between the level of salivary uric acid and all parameters.

\begin{tabular}{ccc}
\hline \multirow{2}{*}{ Correlations } & \multicolumn{2}{c}{ level of salivary uric acid } \\
\cline { 2 - 3 } & $\mathrm{r}$ & P-value \\
\hline PLI & 0.714 & 0.004 \\
GI & 0.881 & 0.017 \\
CI & 0.136 & $0.000^{* *}$ \\
CAL & 0.632 & $0.0001^{\star *}$ \\
\hline
\end{tabular}

PLI: Plaque index, GI: Gingival index, CI: Calculus index, CAL: Clinical attachment loss. ${ }^{* * H i g h l y ~ s t a t i s t i-~}$ cally significant. 
There was high plaque formation in the present study among kidney stones patients more than non-kidney stones patients, and dental calculus is dental plaque mineralization [18]. The result of the present study has supported this clarification that exhibited there was a correlation between the high value of PLI and an increase of calculus formation among kidney stones patients. Consequently, we evaluated the patients depending on the history of kidney stones, clinical examination of periodontal tissues and, assessment of calculus formation on the teeth surfaces with a uric acid level in saliva.

Results of the former study displayed that the prevalence of kidney stones is more among males (66\%) than females and widespread within 31 - 40 years age group [35]. These results are appropriate with the objective of the present study that was done on 120 male Saudi patients within the age group (25 - 70) years, which included the age group $(31-40)$ years. We explained to the patients the nature of calculus and, its impact on oral health and periodontal status moreover the possible correlation between calculus formation and the formation of kidney stones. According to previous studies, many researchers found significant links between kidney stones formation and accumulations of dental calculus on the teeth surfaces [34] [36]. Identical to the clinical findings in this study, we detected that there are significant correlations between kidney stones formation and dental calculus accumulations.

The current study and the study conducted by Brito et al. showed that GI and CAL were more in kidney stones patients group than non-kidney stones patients [37]. Consistent with the clinical findings of other studies revealed that periodontal destruction was more among kidney stones patients more than non-kidney stones patients [17] [38] [39] [40].

The higher mean values of GI, CI, and CAL among study group in the present study may be due to the higher mean values of PLI scores that recorded among kidney stones patients more than non-kidney stones patients in all study groups with statistical significance difference in all clinical parameters except GI among of kidney stones patients and CAL in kidney stones patients and non-kidney stones patients since dental plaque is the main etiological factor of periodontal diseases [41]. Xerostomia represents an oral complication of kidney stones disease and maybe predispose factors to gingival disease [42]. This is in agreement with the present study where the gingival disease was detected among all the kidney stones patients.

On the other hand, Uric acid is a major salivary antioxidant of healthy and diseased periodontal cases and there were many early studies comparing the salivary antioxidants levels among periodontitis patients and their levels in individuals with healthy periodontal tissues [8] [43]. Our results displayed the presence of a higher amount of salivary uric acid level in kidney stones patients compared to non-kidney stones patients. This result is supported by the results of other studies done by Hadi BA et al. (2011) and Xia Y et al. (2012) that displayed the levels of salivary uric acid in kidney stones patients were significantly higher than those of non-kidney stones patients [44] [45]. 
In the present study, there was an association between an increase of periodontal destruction and an increase in the level of salivary uric acid among kidney stones patients compared to non-kidney stones patients. Consequently, the present study demonstrated that there were highly significant differences in the dental calculus mean values among kidney stones patients than the non-kidney stones patients. This may be due to the presence of higher values of salivary uric acid level among kidney stones patients $(\mathrm{mg} / \mathrm{dl})$ compared to non-kidney stones patients in all study groups which corresponds to the results of other studies [46] [47]. Finally, many previous studies have tried to explain the relation between renal diseases in general, and the severity and type of periodontal diseases, but their findings were incompatible, that may be due to the massive debate in periodontal disease classification [48]-[53].

\section{Strength and Limitations}

This study participates in the current efforts to explain the relationship between salivary uric acid levels and periodontal health among Kidney stones patients and non-Kidney stones patients. However, the cross-sectional nature of the study, self-reported medical conditions through medical history in the patient's case sheet, participants' consents and selection of study participants from the patients of a college of dentistry, King Khalid University, absence of female participants, and small sample size considered as limitations of the study.

\section{Conclusion}

We studied the cases depending on the kidney stones' history, dental calculus and periodontal status evaluation and salivary uric acid measurement where there was a statistically significant difference in dental calculus formation among patients with kidney stones more than non-kidney stones patients. This correlation between an increase of dental calculus formation and kidney stones formation in the current study may be due to the minerals that form the kidney stones that may be accumulate in other areas of the body. This may be evidence that dental calculus can form a diagnostic indicator to help in the detection and diagnosis of kidney stones in case of recurrence of dental calculus formation. Consequently, the detection of a dental calculus during the clinical examination may be considered a predictor of kidney stones present, and this conclusion should be subjected to more studies.

\section{Acknowledgements}

The authors would like to thank all the participants in this study and the all staff in dental clinics, college of dentistry, King Khalid University for their help in the collection of data from the patients' files.

\section{Conflicts of Interest}

The authors declare no conflicts of interest regarding the publication of this paper. 


\section{References}

[1] Phillip, D. (2015) The Role of Biofilms in Health and Disease. In: Kenneth, E. and Philip, O., Eds., Practical Periodontics, Elsevier Publishers, London, 51.

[2] Marsh, P.D. (2004) Dental Plaque as a Microbial Biofilm. Caries Research, 38, 204-211. https://doi.org/10.1159/000077756

[3] Miricescu, D., Grebu, M., Totan, A., Didilescu, A. and Radulescu, R. (2011) The Antioxidant Potential of Saliva: Clinical Significance in Oral Diseases. Therapeutics, Pharmacology and Clinical Toxicology, 15, 139-143.

[4] Peter, S. (2004) Epidemiology Etiology and Prevention of Dental Caries. In: Peter, S., Ed., Essential of Preventive and Community Dentistry, 2nd Edition, Arya (Medi) Publishing House, New Delhi, 250-255.

[5] Novakovic, N., Todorovic, T., Rakic, M., Milinkovic, I., Dozic, I., Jankovic, S., et al. (2014) Salivary Antioxidants as Periodontal Biomarkers in Evaluation of Tissue Status and Treatment Outcome. Journal of Periodontal Research, 49, 129-136. https://doi.org/10.1111/jre.12088

[6] Dahiya, P., Kamal, R., Gupta, R., Bhardwaj, R., Chaudhary, K. and Kaur, S. (2013) Reactive Oxygen Species in Periodontitis. Journal of Indian Society of Periodontology, 17, 411-416. https://doi.org/10.4103/0972-124X.118306

[7] Lippi, G., Montagnana, M., Franchini, M., Favaloro, E.J. and Targher, G. (2008) The Paradoxical Relationship between Serum Uric Acid and Cardiovascular Disease. Clinica Chimica Acta, 392, 1-7. https://doi.org/10.1016/j.cca.2008.02.024

[8] Moore, S., Calder, K.A., Miller, N.J. and Rice-Evans, C.A. (1994) Antioxidant Activity of Saliva and Periodontal Disease. Free Radical Research, 21, 417-425. https://doi.org/10.3109/10715769409056594

[9] Nagler, R.M., Klein, I., Zarzhevsky, N., Drigues, N. and Reznick, A.Z. (2002) Characterization of the Differentiated Antioxidant Profile of Human Saliva. Free Radical Biology and Medicine, 32, 268-277. https://doi.org/10.1016/S0891-5849(01)00806-1

[10] Jenifer, H.D., Bhola, S., Kalburgi, V., Warad, S. and Kokatnur, V.M. (2015) The Influence of Cigarette Smoking on Blood and Salivary Super Oxide Dismutase Enzyme Levels among Smokers and Nonsmokers-A Cross Sectional Study. Journal of Traditional and Complementary Medicine, 5, 100-105. https://doi.org/10.1016/j.jtcme.2014.11.003

[11] Friedlander, J.I., Moreira, D.M., Hartman, C., et al. (2014) Comparison of the Metabolic Profile of Mixed Calcium Oxalate/Uric Acid Stone Formers to That of Pure Calcium Oxalate and Pure Uric Acid Stone Formers. Urology, 84, 289-294. https://doi.org/10.1016/j.urology.2014.04.019

[12] Reed, B. and Gitomer, W. (2007) The Genetics of Stone Disease. In: Stoller, M. and Meng, M., Eds., Urinary Stone Disease, Humena Press, Totowa, 35. https://doi.org/10.1007/978-1-59259-972-1 3

[13] Pearle, M. and Lotan, Y. (2007) Urinary Lithiasis. In: Wein, A., Kavoussi, L., Novick, A., Partin, A. and Peters, C., Eds., Campbell-Walsh Urology, 9th Edition, Saunders, Philadelphia, 1363-1392.

[14] Michel, D., Dominique, B. and Emmanuel, L. (2015) Randall's Plaque as the Origin of Calcium Oxalate Kidney Stones. Urolithiasis, 43, 5-11. https://doi.org/10.1007/s00240-014-0703-y

[15] Reilly, R. (2005) The Patient with Kidney Stones. In: Schrier, R., Ed., Manual of Nephrology, 6th Edition, Lippincott Williams and Wilkins, Philadelphia, 79-91. 
[16] El-Faqih, S.R. (2012) Epidemiology of Stone Disease in Saudi Arabia with Overview of the Regional Differences. In: Urolithiasis, Springer-Verlag, London, 77-83. https://doi.org/10.1007/978-1-4471-4387-1 10

[17] Tadakamadla, J., Kumar, S. and Mamatha, G.P. (2014) Comparative Evaluation of Oral Health Status of Chronic Kidney Disease (CKD) Patients in Various Stages and Healthy Controls. Special Care in Dentistry, 34, 122-126. https://doi.org/10.1111/scd.12040

[18] Hinrichs, J. (2002) The Role of Dental Calculus and Other Predisposing Factors. In: Newman, M., Takei, H. and Carranza, F., Eds., Carranza's Clinical Periodontology, 9th Edition, Saunders Company, Philadelphia, 182-187.

[19] Sargolzaee, N., Najafi, M., Mohammad zadeh, M., Taheri, M. and Okati, B. (2007) The Evaluation of Calculus Rate in Patients with Nephrolithiasis. Journal of Mashhad Dental School, 31, 195-200.

[20] Lang, N., Mombelli, A. and Attstrom, R. (2008) Oral Biofilm and Calculus. In: Lindhe, J., Lang, N. and Karring, T., Eds., Clinical Periodontology and Implant Dentistry, 5th Edition, Blackwell Munksgaard, Oxford, 197-202.

[21] Stoller, M.L. (2008) Urinary Stone Disease. In: Tanagho, E.A. and McAninch, J.W., Eds., Smith's General Urology, 17th Edition, McGraw Hill, New York, 246.

[22] Faul, F., Erdfelder, E., Lang, A.G. and Buchner, A. (2007) G*Power 3: A Flexible Statistical Power Analysis Program for the Social, Behavioral, and Biomedical Sciences. Behavior Research Methods, 39, 175-191. https://doi.org/10.3758/BF03193146

[23] Silness, J. and Loe, H. (1964) Periodontal Disease in Pregnancy II. Acta Odontologica Scandinavica, 22, 747-759. https://doi.org/10.3109/00016356408993968

[24] Loe, H. and Silness, J. (1963) Periodontal Disease in Pregnancy I. Acta Odontologica Scandinavica, 21, 533-551. https://doi.org/10.3109/00016356309011240

[25] Greene, J.C. and Vermillion, J.R. (1964) The Simplified Oral Hygiene Index. Journal of the American Dental Association, 68, 7-13. https://doi.org/10.14219/jada.archive.1964.0034

[26] Chapple, I.L.C., Mealey, B.L., et al. (2018) Periodontal Health and Gingival Diseases and Conditions on an Intact and a Reduced Periodontium: Consensus Report of Workgroup 1 of the 2017 World Workshop on the Classification of Periodontal and Peri-Implant Diseases and Conditions. Journal of Clinical Periodontology, 45, S68-S77.

[27] Caton, J., Armitage, G., Berglundh, T., et al. (2018) A New Classification Scheme for Periodontal and Periimplant Diseases and Conditions-Introduction and Key Changes from the 1999 Classification. Journal of Clinical Periodontology, 45, S1-S8. https://doi.org/10.1111/jcpe.12935

[28] Papapanou, P.N., Sanz, M., et al. (2018) Periodontitis: Consensus Report of Workgroup 2 of the 2017 World Workshop on the Classification of Periodontal and Peri-Implant Diseases and Conditions. Journal of Clinical Periodontology, 45, S162-S170.

[29] Navazesh, M. and Kumar, S.K. (2008) University of Southern California School of Dentistry. Measuring Salivary Flow: Challenges and Opportunities. Journal of the American Dental Association, 139, 35S-40S. https://doi.org/10.14219/jada.archive.2008.0353

[30] Avogaro, A. and Fadini, G.P. (2015) Mechanisms of Ectopic Calcification: Implications for Diabetic Vasculopathy. Cardiovascular Diagnosis and Therapy, 5, 343-352. 
[31] Valenzuela, A. and Chung, L. (2015) Calcinosis: Pathophysiology and Management. Current Opinion in Rheumatology, 27, 542-548. https://doi.org/10.1097/BOR.0000000000000220

[32] Aguilar-Ruiz, J., Arrabal-Polo, M.A., Sierra, M. and Arrabal-Martin, M. (2012) Application of Mineralogical Techniques in the Study of Human Lithiasis. Ultrastructural Pathology, 36, 367-376. https://doi.org/10.3109/01913123.2012.729879

[33] Maiuolo, J., Oppedisano, F., Gratteri, S., Muscoli, C. and Mollace, V. (2016) Regulation of Uric Acid Metabolism and Excretion. International Journal of Cardiology, 213, 8-14. https://doi.org/10.1016/j.ijcard.2015.08.109

[34] Davidovich, E., Davidovits, M., Peretz, B., et al. (2009) The Correlation between Dental Calculus and Disturbed Mineral Metabolism in Paediatric Patients with Chronic Kidney Disease. Nephrology Dialysis Transplantation, 24, 2439-2445. https://doi.org/10.1093/ndt/gfp101

[35] Patel, A.C. and Mehta, N.H. (2014) Epidemiological Characteristics of Renal Stone Patients Age (21-60) and Barriers in Their Dietary Modification in Saurashtra Region. International Journal of Research in Medical Sciences, 2, 80-83. https://doi.org/10.5455/2320-6012.ijrms20140216

[36] Shaimaa, K.Y. and Mohammed, S.A. (2012) Dental Calculus in Relation to Idiopathic Calcium Renal Stone. Journal of Baghdad College of Dentistry, 24, 140-145.

[37] Brito, F., Almeida, S., Figueredo, C., Bregman, R., Suassuna, J. and Fischer, R. (2012) Extent and Severity of Chronic Periodontitis in Chronic Kidney Disease Patients. Journal of Periodontal Research, 47, 426-430. https://doi.org/10.1111/j.1600-0765.2011.01449.x

[38] Nylund, K., Meurman, J., Heikkinen, A., Honkanen, E., Vesterinen, M. and Ruokonen, H. (2015) Oral Health in Predialysis Patients with Emphasis on Periodontal Disease. Quintessence International, 46, 899-907.

[39] Tawig, A., Jamal, B., Eskandrani, R., Al Dayel, L., Al Twaijry, S., Al Ghamdi, E., et al. (2016) Assessment of Periodontal Disease Severity among Patients at Different Stages of Chronic Kidney Disease. Journal of International Oral Health, 8, 307-312.

[40] Sharma, P., Dietrich, T., Ferro, C., Cockwell, P. and Chapple, I. (2016) Association between Periodontitis and Mortality in Stages 3-5 Chronic Kidney Disease: NHANES III and Linked Mortality Study. Journal of Clinical Periodontology, 43, 104-113. https://doi.org/10.1111/jcpe.12502

[41] Gao, X., Deng, D. and Geng, Q.A. (2000) Study of Oral Health Condition in Individuals with No Oral Hygiene and Its Association with Plaque Acidogenesis. Chinese Journal of Dental Research, 3, 44-48.

[42] Porter, S.R., Scully, C. and Hegarty, A.M. (2004) An Update of the Etiology and Management of Xerostomia. Oral Surgery, Oral Medicine, Oral Pathology, Oral Radiology, and Endodontology, 97, 28-46. https://doi.org/10.1016/j.tripleo.2003.07.010

[43] Akalin, F.A., Baltacioglu, E., Alver, A. and Karabulut, E. (2007) Lipid Peroxidation Levels and Total Oxidant Status in Serum, Saliva and Gingival Crevicular Fluid in Patients with Chronic Periodontitis. Journal of Clinical Periodontology, 34, 558-565. https://doi.org/10.1111/j.1600-051X.2007.01091.X

[44] Hadi, B.A. and Al-Jubouri, R.H. (2011) Salivary and Plasmaanalysis of Oxidative Stress Biomarkers in Endstage Renal Failure Patients. Journal of Baghdad College of Dentistry, 23, 46-50.

[45] Xia, Y., Peng, C., Zhou, Z., Cheng, P., Sun, L., Peng, Y. and Xiao, P. (2012) Clinical Significance of Saliva Urea, Creatinine, and Uric Acid Levels in Patients with 
Chronic Kidney Disease. Journal of Central South University. Medical Sciences, 37, 1171-1176.

[46] Brock, G.R., Butterworth, C.J., Matthews, J.B. and Chapple, I.L. (2004) Local and Systemic Total Antioxidant Capacity in Periodontitis and Health. Journal of Clinical Periodontology, 31, 515-521. https://doi.org/10.1111/j.1600-051X.2004.00509.x

[47] Tsai, C.C., Chen, H.S., Chen, S.L., Ho, Y.P., Ho, K.Y., Wu, Y.M., et al. (2005) Lipidperoxidation: A Possible Role in the Induction and Progression of Chronic Periodontitis. Journal of Periodontal Research, 40, 378-384. https://doi.org/10.1111/j.1600-0765.2005.00818.x

[48] Bayraktar, G., Kurtulus, I., Duraduryan, A., et al. (2007) Dental and Periodontal Findings in Hemodialysis Patients. Oral Diseases, 13, 393-397.

https://doi.org/10.1111/j.1601-0825.2006.01297.x

[49] Sakallıglu, E.E., Lutfioglu, O.M., Ozkaya, E., Aliyev, G., Acıkgoz and Firatlı, E. (2007) Fluid Dynamics of Gingiva and Gingival Health in Children with End Stage Renal Failure. Archives of Oral Biology, 52, 1194-1199.

https://doi.org/10.1016/j.archoralbio.2007.05.004

[50] Bayraktar, G., Kurtulus, R.I., Kazancioglu, et al. (2008) Evaluation of Periodontal Parameters in Patients Undergoing Peritoneal Dialysis or Hemodialysis. Oral Diseases, 14, 185-189. https://doi.org/10.1111/j.1601-0825.2007.01372.x

[51] Davidovich, E., Schwarz, Z., Davidovitch, M., Eidelman, E. and Bimstein, E. (2005) Oral Findings and Periodontal Status in Children, Adolescents and Young Adults Suffering from Renal Failure. Journal of Clinical Periodontology, 32, 1076-1082. https://doi.org/10.1111/j.1600-051X.2005.00812.X

[52] Fang, F., Wu, B., Qu, Q., et al. (2015) The Clinical Response and Systemic Effects of Non-Surgical Periodontal Therapy in End-Stage Renal Disease Patients: A 6-Month Randomized Controlled Clinical Trial. Journal of Clinical Periodontology, 42, 537-546. https://doi.org/10.1111/jcpe.12411

[53] Almeida, S., Figueredo, C.M., Lemos, C., Bregman, R. and Fischer, R.G. (2017) Periodontal Treatment in Patients with Chronic Kidney Disease: A Pilot Study. Journal of Periodontal Research, 52, 262-267. https://doi.org/10.1111/jre.12390 